\title{
Use of Remote Sensing and GIS Techniques in Identification of Landslide Vulnerable Zones of Shastri River Basin Along the West Coast of Ratnagiri District, Maharashtra
}

\author{
S. B. Joshi $* \dagger$ and D. D. Kulkarni** \\ *Department of Geology, Walchand College of Arts \& Science, Solapur, Maharashtra, India \\ **Department of Geology, School of Earth Sciences, P.A.H. Solapur University, Maharashtra, India \\ $\dagger$ Corresponding author: S. B. Joshi; sanjaybj65@ rediffmail.com
}

Nat. Env. \& Poll. Tech.

Website: www.neptjournal.com

Received: 08-06-2021

Revised: $09-07-2021$

Accepted: 14-07-2021

Key Words:

Natural hazards

Remote sensing

DEM

GIS

Landslide

\begin{abstract}
The atmosphere, hydrosphere, and lithosphere are subjected to different processes, leading to natural hazards like weathering, erosion, floods, cyclones, landslides, earthquakes, tectonic movements, etc. Environmental degradation is a serious aspect of the recent past, mainly due to natural and manmade interactions. The pressure for infrastructure development due to rapid urbanization has led to the expansion of construction activities. It has catapulted the frequency of landslides to dramatic proportions in recent decades, especially along western ghats. The West Coast of India (WCI) has attracted the attention of Geo-scientists due to its neo-tectonic setup, continuing seismic activities, sea-level changes, and also due to environmental degradation. It is followed that very limited attempts have been made related to the land sliding along the west coast tract of Maharashtra. The present investigations are emphasized mainly to locate the landslide vulnerable zones of Shastri River Basin (SRB), Ratnagiri district of Maharashtra by using remote sensing data, GIS techniques along field studies. The area lies within a triple junction of Koyana-Kurduwadi Lineament (KKL), West Coast Fault (WCF), and Panvel Flexure (PF). Based on the integration of data from various thematic maps viz. lithology, lineaments, slope, geomorphology, land use-land cover along with inventory map, Landslide Vulnerable Map (LVM) of the SRB has been prepared. It follows that about $29 \%$ area of the SRB forms a highly vulnerable zone for land sliding. These zones are mainly confined to steep slopes, wasteland, highly weathered basalts, and deep valleys and in the vicinity of lineaments.
\end{abstract}

\section{INTRODUCTION}

Land degradation is one of the serious environmental problems, causing due to the demand for natural resources by the growing population for food, fodder, fuelwood, and intensive industrial as well as anthropogenic activities. The key problems of land degradation are desertification, deforestation, soil erosion, waterlogging, salination, economic pressure, and poverty. Due to natural and manmade interactions, the atmosphere, hydrosphere, and lithosphere are subjected to different processes, leading to natural hazards. Natural hazards and disasters continue to have an increasing impact on humans around the world. However, studies show that this impact is heavily tilted towards developing countries like India, which might be due to the increasing population. Amongst all the natural disasters, landslides severely damage infrastructure, cause a loss of life and properties, and impact the daily life of people living in the affected regions (Juang et al. 2019). The pressure for infrastructure development due to rapid urbanization has led to the expansion of construction activities. It has accelerated the frequency of landslides to dramatic proportions in recent decades, especially in hilly terrains.

The studies carried out by many scholars have indicated that the various geosystems viz. lithology, lineaments, geomorphology, slope, land use/land cover, etc. occurring in different combinations, assign differing landslide vulnerability grades (Nagarajan et al. 1998, Guzzetti et al. 2012, Meena et al. 2019, Prakash et al. 2020, Edison \& Ganpati 2020). Ramakrishnan et al. (2002) prepared thematic layers in a GIS platform using aerial photos and orthophotos. Heavy rainfall is a critical factor in triggering landslides as it generates a rapid increase in pore pressure in the vadose zone and groundwater flow in the saturated area (Jiu et al. 2005). Various geological structures along with lineaments and lithology play a vital role in triggering landslides (Greenbaum et al. 1995). The susceptibility of the slopes for landslides can also be influenced by land-use and landcover changes caused due to natural or manmade activities (Diaz et al. 2005). According to Van Westen et al. (2006), the soil also acts as one of the most sensitive parameters of land sliding. 
The pre- and post-disaster landslide studies have been carried out by many governments and semi-government agencies, academic institutions viz. GSI, CBRI, CRRI, NRSA, WIHG, DTRL, Govind Ballabh Pant Institute of Himalayan Environment, IIT-Roorkee, etc. These organizations have carried work mostly related to landslide-prone regions of the parts of the Himalayas, Uttarakhand, and most of the North-Eastern parts of India. However, very limited attempts have been made with respect to land sliding along Western escarpments of India, especially the west coast of Maharashtra. Therefore, the present studies have been envisaged to locate the landslide-prone zones present within the Shastri River Basin (SRB), Ratnagiri district of Maharashtra. The SRB lies within a triple junction of Koyana- Kurduwadi Lineament (KKL), West Coast Fault (WCF), and Panvel Flexure (PF). It forms a part of one of the seismically active regions of the Indian continent (Valdiya 2011), characterized by sloping topography, presence of shear zones, accelerating rain-splash erosion, sheet erosion as well as gully erosion.

\section{OBJECTIVES}

The main objectives of the present study of SRB are to

- Investigate the causative factors of landslides.

- Prepare various thematic maps of these factors viz. slope, geology, lineament, geomorphology, land use/ land cover, etc.

- Prepare a map of the landslide vulnerable zones of SRB.

\section{STUDY AREA}

The area lies along the West Coast of Maharashtra (India)

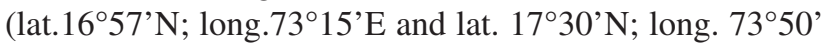
E) (Figs. 1 and 2). Shastri is a seventh-ordered river having a length of about $72 \mathrm{~km}$. It flows from NE to SW direction following major trends of the lineaments and covers an area of about $2098 \mathrm{~km}^{2}$ (SOI topographic sheet Nos. 47 G/3, G/4,

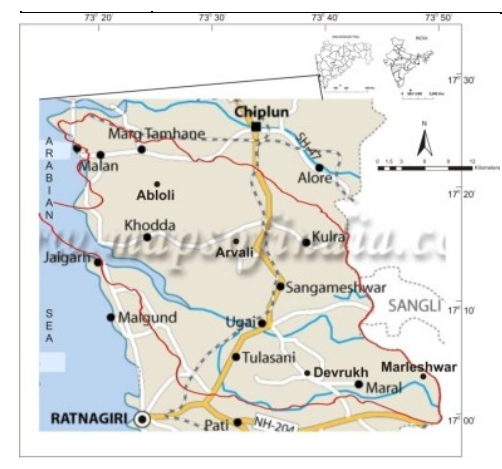

Fig. 1: Location map of the area (Shastri River Basin).
G/7, G/8, G/11, G/12, and 47 H/9). The SRB forms a part of the western periphery of the Deccan Trap province of India. It represents the presence of basaltic lava flows of Cretaceous to Eocene age (Mitchell \& Cox 1988), exposed along river valleys, valley sides, and near shore. Most of these are capped by laterites of the Pleistocene age. The drainage pattern is dendritic to sub-dendritic, with trellis and sub-parallel at some places controlled by lineaments.

\section{MATERIALS AND METHODS}

Present investigations have been carried through the following stages (Fig. 3).

Pre-field studies: These include the review of literature related to landslides, collection of data from topographic sheets, and remote sensing data

Field studies: These include identification of landslides in the field; a collection of their coordinates using GPS, a collection of attributes and training data (GCP) for supervised classification of remote sensing data, and identification of various types of anthropogenic activities in the study region. The GPS data is also collected for the ground truth,

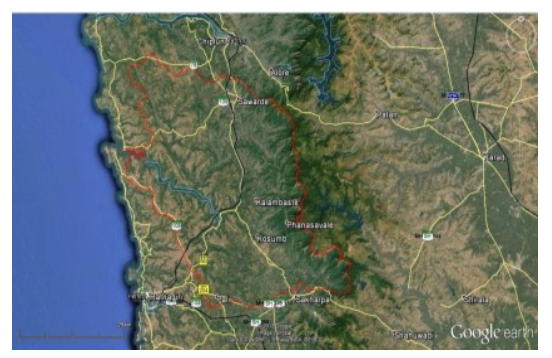

Fig. 2: Map of the SRB (Google Image).

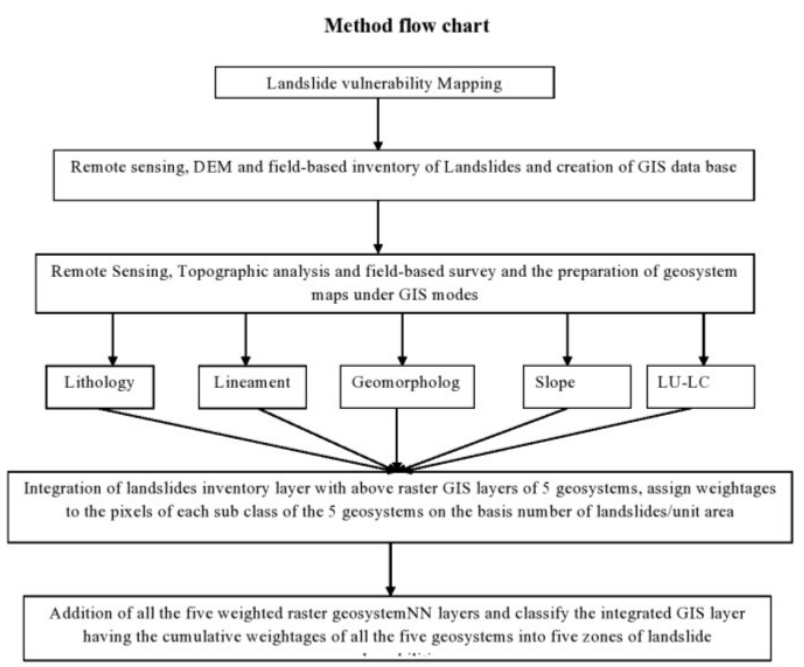

Fig. 3: Method flow chart. 
Table 1: Distribution and the morphology of the existing landslides in SRB.

\begin{tabular}{|c|c|c|c|c|c|c|c|c|}
\hline $\begin{array}{l}\text { Location } \\
\text { No. }\end{array}$ & L. S. -1 & L. S. -2 & L. S. -3 & L. S. -4 & L. S. -5 & L. S. -6 & L. S. -7 & L. S. -8 \\
\hline Location & Bhatgaon & Bhatgaon & $\begin{array}{l}\text { Near Ukshi } \\
\text { village }\end{array}$ & $\begin{array}{l}\text { Close to } \\
\text { Konkan } \\
\text { Railway } \\
\text { line }\end{array}$ & Karjuve Bridge & $\begin{array}{l}\text { Near Bhat- } \\
\text { gaon village }\end{array}$ & $\begin{array}{l}\text { Near } \\
\text { Bhatgaon } \\
\text { village }\end{array}$ & $\begin{array}{l}\text { Near Bhuiwadi } \\
\text { village }\end{array}$ \\
\hline Road Status & $\begin{array}{l}\text { Bhat- } \\
\text { gaon-Ablo- } \\
\text { li Road }\end{array}$ & $\begin{array}{l}\text { Bhat- } \\
\text { gaon-Abloli } \\
\text { Road }\end{array}$ & $\begin{array}{l}\text { Jaigarh-Uk- } \\
\text { shi Road }\end{array}$ & $\begin{array}{l}\text { Phun- } \\
\text { gus-Sang- } \\
\text { meshwar } \\
\text { Road }\end{array}$ & $\begin{array}{l}\text { Phungus-Sang- } \\
\text { meshwar Road }\end{array}$ & $\begin{array}{l}\text { Bhat- } \\
\text { gaon-Asore } \\
\text { Road }\end{array}$ & $\begin{array}{l}\text { Bhat- } \\
\text { gaon-Asore } \\
\text { Road }\end{array}$ & $\begin{array}{l}\text { Narsinge-Bhui- } \\
\text { wadi Road }\end{array}$ \\
\hline $\begin{array}{l}\text { Lat. And } \\
\text { Long. }\end{array}$ & $\begin{array}{l}17^{\circ} 18^{\prime} 20^{\prime \prime}, \\
73^{\circ} 21^{\prime} 33^{\prime \prime}\end{array}$ & $\begin{array}{l}17^{\circ} 17^{\prime} 54^{\prime \prime}, \\
73^{\circ} 21^{\prime} 45^{\prime \prime}\end{array}$ & $\begin{array}{l}17^{\circ} 06^{\prime} 35^{\prime \prime}, \\
73^{\circ} 26^{\prime} 30^{\prime \prime}\end{array}$ & 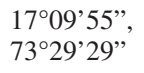 & $\begin{array}{l}17^{\circ} 11^{\prime} 15^{\prime \prime}, \\
73^{\circ} 28^{\prime} 22^{\prime \prime}\end{array}$ & $\begin{array}{l}17^{\circ} 12^{\prime} 26^{\prime \prime}, \\
73^{\circ} 25^{\prime} 00^{\prime \prime}\end{array}$ & $\begin{array}{l}\text { 17²11'46", } \\
73^{\circ} 25^{\prime} 10^{\prime \prime}\end{array}$ & $\begin{array}{l}17^{\circ} 12^{\prime} 40^{\prime \prime}, \\
73^{\circ} 24^{\prime} 00^{\prime \prime}\end{array}$ \\
\hline Altitude & $220 \mathrm{~m}$ & $120 \mathrm{~m}$ & $210 \mathrm{~m}$ & $100 \mathrm{~m}$ & $100 \mathrm{~m}$ & $140 \mathrm{~m}$ & $180 \mathrm{~m}$ & $200 \mathrm{~m}$ \\
\hline $\begin{array}{l}\text { Nature of } \\
\text { Slope }\end{array}$ & $\begin{array}{l}\text { Naturally } \\
\text { Moderately } \\
\text { Steep }\end{array}$ & $\begin{array}{l}\text { Naturally } \\
\text { Moderately } \\
\text { Steep }\end{array}$ & $\begin{array}{l}\text { Naturally } \\
\text { Moderate } \\
\text { Top cut } \\
\text { Slope }\end{array}$ & $\begin{array}{l}\text { Naturally } \\
\text { Moderate } \\
\text { Top cut } \\
\text { Slope }\end{array}$ & $\begin{array}{l}\text { Natural Top cut } \\
\text { Slope }\end{array}$ & $\begin{array}{l}\text { Naturally } \\
\text { Moderate } \\
\text { Top Cut } \\
\text { Slope }\end{array}$ & $\begin{array}{l}\text { Naturally } \\
\text { Top cut } \\
\text { Slope (Hill } \\
\text { Side) }\end{array}$ & $\begin{array}{l}\text { Naturally Steep } \\
\text { Top Cut Slope }\end{array}$ \\
\hline $\begin{array}{l}\text { Strike Dir. } \\
\text { of Hill }\end{array}$ & NNW-SSE & NNW-SSE & $\mathrm{N}-\mathrm{S}$ & NNE-SSW & NNE -SSW & NNW-SSE & $\mathrm{N}-\mathrm{S}$ & $\mathrm{N}-\mathrm{S}$ \\
\hline Rock Type & $\begin{array}{l}\text { Highly } \\
\text { altered } \\
\text { Deccan } \\
\text { Basalt }\end{array}$ & $\begin{array}{l}\text { Lateritic } \\
\text { rock mass }\end{array}$ & $\begin{array}{l}\text { Highly } \\
\text { Jointed } \\
\text { Deccan } \\
\text { Basalt }\end{array}$ & $\begin{array}{l}\text { Lateritic } \\
\text { soil }\end{array}$ & $\begin{array}{l}\text { Highly altered } \\
\text { Laterite }\end{array}$ & $\begin{array}{l}\text { Highly } \\
\text { weathered, } \\
\text { porphyritic } \\
\text { Basalt }\end{array}$ & $\begin{array}{l}\text { Highly } \\
\text { weathered } \\
\text { Lateritic } \\
\text { Soil }\end{array}$ & $\begin{array}{l}\text { Weathered } \\
\text { Basalt }\end{array}$ \\
\hline Soil Type & $\begin{array}{l}\text { Fine } \\
\text { Yellowish } \\
\text { to Brown, } \\
3 \text { m thick }\end{array}$ & $\begin{array}{l}\text { Dark Brown, } \\
1 \mathrm{~m} \text { thick }\end{array}$ & $\begin{array}{l}\text { Light } \\
\text { yellow thin } \\
<0.5 \mathrm{~m}\end{array}$ & $\begin{array}{l}\text { Deep red, } \\
\text { Depth } \\
\text { couldn't } \\
\text { ascertain }\end{array}$ & $\begin{array}{l}\text { Dark brown } \\
\text { Lateritic Soil, } \\
\text { upto } 4 \mathrm{~m} \text { thick }\end{array}$ & $\begin{array}{l}\text { Brown, thin } \\
<0.5 \mathrm{~m}\end{array}$ & $\begin{array}{l}\text { Brown, thin } \\
2-5 \mathrm{~m}\end{array}$ & $\begin{array}{l}\text { Brown, } 4-5 \text { m } \\
\text { thick }\end{array}$ \\
\hline $\begin{array}{l}\text { Type of } \\
\text { L. S. }\end{array}$ & $\begin{array}{l}\text { Debris } \\
\text { Slide }\end{array}$ & Minor Slump & Rock Fall & Soil slump & Soil Slump & Rock Slide & Soil Slump & Soil Slump \\
\hline $\begin{array}{l}\text { L. S. Di- } \\
\text { mensions }\end{array}$ & $\begin{array}{l}\text { Ht. of } \\
\text { Crown } \\
\approx 10 \mathrm{~m}, \\
\text { Width } \approx \\
4 \mathrm{~m}\end{array}$ & $\begin{array}{l}\text { Ht. of Crown } \\
\approx 3.5 \mathrm{~m}, \\
\text { Width } \approx 7 \mathrm{~m}\end{array}$ & $\begin{array}{l}\text { Ht. of } \\
\text { Crown } \approx \\
2-4 \mathrm{~m}, \\
\text { Width } \approx \\
60 \mathrm{~m}\end{array}$ & $\begin{array}{l}\text { Ht. of } \\
\text { Crown } \\
\approx 2.5 \mathrm{~m} \text {, } \\
\text { Width } \\
70 \mathrm{~m}\end{array}$ & $\begin{array}{l}\text { Ht. of Crown } \\
\approx 4 \mathrm{~m} \text {, Width } \approx \\
52 \mathrm{~m}\end{array}$ & $\begin{array}{l}\text { Ht. of } \\
\text { Crown } \approx 6 \\
\mathrm{~m}, \text { Width } \approx \\
85 \mathrm{~m}\end{array}$ & $\begin{array}{l}\text { Ht. of } \\
\text { Crown } \approx 3 \\
\mathrm{~m}, \text { Width } \approx \\
40 \mathrm{~m}\end{array}$ & $\begin{array}{l}\text { Ht. of Crown } \\
\approx 4 \mathrm{~m} \text {, Width } \approx \\
25 \mathrm{~m}\end{array}$ \\
\hline
\end{tabular}

\begin{tabular}{|c|c|c|c|c|c|c|}
\hline Location No. & L. S. -9 & L. S. -10 & L. S. -11 & L. S. -13 & L. S. -14 & L. S. -15 \\
\hline Location & $\begin{array}{l}\text { Near Bhuiwadi } \\
\text { village }\end{array}$ & $\begin{array}{l}\text { Near Bhuiwadi } \\
\text { village }\end{array}$ & $\begin{array}{l}\text { Near Dhamnase } \\
\text { village }\end{array}$ & Near Kondye village & $\begin{array}{l}\text { Near Chavanwadi } \\
\text { village }\end{array}$ & $\begin{array}{l}\text { Near Nayari vil- } \\
\text { lage (Hilltop) }\end{array}$ \\
\hline Road Status & $\begin{array}{l}\text { Chaphe - Agar- } \\
\text { naral - Bhiwandi } \\
\text { Road }\end{array}$ & $\begin{array}{l}\text { Khalgaon - } \\
\text { Bhuiwadi Road }\end{array}$ & $\begin{array}{l}\text { Ratnagiri - Gan- } \\
\text { patipule Road }\end{array}$ & $\begin{array}{l}\text { Close to Sangmesh- } \\
\text { war - Ratnagiri Road }\end{array}$ & $\begin{array}{l}\text { Devrukh - Sang- } \\
\text { meshwar Road }\end{array}$ & $\begin{array}{l}\text { Nayari - Sang- } \\
\text { meshwar Road }\end{array}$ \\
\hline $\begin{array}{l}\text { Lat. and } \\
\text { Long. }\end{array}$ & $\begin{array}{l}\text { 17'13'14", } \\
73^{\circ} 20^{\prime} 15^{\prime \prime}\end{array}$ & $\begin{array}{l}17^{\circ} 12^{\prime} 48^{\prime \prime}, \\
73^{\circ} 23^{\prime} 21^{\prime \prime}\end{array}$ & $\begin{array}{l}\text { 1707'55", } \\
73^{\circ} 19^{\prime} 31^{\prime \prime}\end{array}$ & 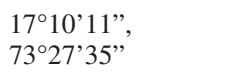 & $\begin{array}{l}\text { 1707’04”, } \\
\text { 73³2’32”' }\end{array}$ & $\begin{array}{l}17^{\circ} 12^{\prime} 55^{\prime \prime}, \\
73^{\circ} 39^{\prime} 11^{\prime \prime}\end{array}$ \\
\hline Altitude & $200 \mathrm{~m}$ & $180 \mathrm{~m}$ & $160 \mathrm{~m}$ & $200 \mathrm{~m}$ & $200 \mathrm{~m}$ & $300 \mathrm{~m}$ \\
\hline $\begin{array}{l}\text { Nature of } \\
\text { Slope }\end{array}$ & $\begin{array}{l}\text { Naturally Small } \\
\text { cut Slope }\end{array}$ & $\begin{array}{l}\text { Natural Mod- } \\
\text { erate Top Cut } \\
\text { Slope }\end{array}$ & $\begin{array}{l}\text { Natural Top cut } \\
\text { Slope (Hillside) }\end{array}$ & $\begin{array}{l}\text { Naturally Moderate } \\
\text { cut Slope }\end{array}$ & $\begin{array}{l}\text { Natural Moderately } \\
\text { cut Slope (Hillside) }\end{array}$ & $\begin{array}{l}\text { Natural Steep } \\
\text { Slope }\end{array}$ \\
\hline $\begin{array}{l}\text { Strike Dir. } \\
\text { Of Hill }\end{array}$ & $\mathrm{N}-\mathrm{S}$ & NE-SW & NW-SE & $\mathrm{N}-\mathrm{S}$ & NNE-SSW & $\mathrm{N}-\mathrm{S}$ \\
\hline Rock Type & $\begin{array}{l}\text { Deccan Basalt } \\
\text { with } 3 \text { sets of } \\
\text { joints }\end{array}$ & $\begin{array}{l}\text { Soil with Col- } \\
\text { luvial Material }\end{array}$ & Lateritic Blocks & $\begin{array}{l}\text { Laterite, Jointed } \\
\text { Basalt at Base }\end{array}$ & Laterite & $\begin{array}{l}\text { Poorly jointed } \\
\text { Basalt }\end{array}$ \\
\hline Soil Type & $\begin{array}{l}\text { Dark Brown, } 1.5 \\
\text { m thick }\end{array}$ & $\begin{array}{l}\text { Lateritic Red, } \\
4-5 \text { m thick }\end{array}$ & Lateritic Red Soil & $\begin{array}{l}\text { Red Lateritic, } 3 \mathrm{~m} \\
\text { Thick }\end{array}$ & Red, $5 \mathrm{~m}$ thick & $\begin{array}{l}\text { Yellow, Murum, < } \\
0.5 \mathrm{~m} \text { thick }\end{array}$ \\
\hline $\begin{array}{l}\text { Type of } \\
\text { L. S. }\end{array}$ & Rock Fall & Debris Fall & Soil Creep & Debris Slide & $\begin{array}{l}\text { Moderate Soil } \\
\text { Slump }\end{array}$ & $\begin{array}{l}\text { Debris Fall, Cracks } \\
\text { to ground }\end{array}$ \\
\hline $\begin{array}{l}\text { L. S. Dimen- } \\
\text { sions }\end{array}$ & $\begin{array}{l}\text { Ht. of Crown } \approx \\
3.5 \mathrm{~m}, \text { Width } \approx \\
25 \mathrm{~m}\end{array}$ & $\begin{array}{l}\text { Ht. of Crown } \\
\approx 9 \mathrm{~m}, \text { Width } \approx \\
15 \mathrm{~m}\end{array}$ & $\begin{array}{l}\text { Ht. of Crown } \approx \\
3-4 \mathrm{~m}, \text { Width } \\
\approx 9 \mathrm{~m}\end{array}$ & $\begin{array}{l}\text { Ht. of Crown } \approx 5 \mathrm{~m}, \\
\text { Width } \approx 50 \mathrm{~m}\end{array}$ & $\begin{array}{l}\text { Ht. of Crown } \approx 8 \\
\text { m, Width } \approx 60 \mathrm{~m}\end{array}$ & $\begin{array}{l}\text { Ht. of Crown } \approx 10 \\
\text { m, Width } \approx 25 \mathrm{~m}\end{array}$ \\
\hline
\end{tabular}


validation, and accuracy assessment of multispectral and elevation data.

Post-field studies: Post-field study was carried out after preparing thematic maps for validation and accuracy assessment of LVZ using techniques such as Geo-referencing of maps and images; digitization of topographic maps; supervised classification of remote sensing data; generation of Digital Elevation Model (DEM); preparation of various thematic maps viz. slope, geological, lineament, land use/landcover, etc. and preparation of final Landslide Vulnerable Zone (LVZ) map of SRB using ArcGIS techniques.

\section{Data Used}

For the comprehensive study and to achieve more accurate results, following types of data have been used.

- Topographic sheets of 1:50000 scale to create GIS-based vector layers

- IRS-R2 LISS III remote sensing data for temporal and spatial changes and also to prepare various thematic maps in raster format

- Digital Elevated Model (DEM) (Fig. 4)

- Rainfall data from GSDA, Ratnagiri, Govt. of Maharashtra

- IDRISI 3 software for image raster analysis and Arc GIS software for vector analysis.

Attempts have been made to generate a landslide vulnerability map for the study area using a GIS-based geosystem response model.

\section{Location and Characters of Existing Landslides}

In the first step, distribution and the morphology of the existing landslides observed during the field inventories with GPS and data have been presented in (Table 1). The preliminary inventory map has been prepared (Fig. 5).

\section{Generation of GIS Databases on Geosystem Parameters}

Then vector GIS databases showing the features (in the form of polygons) were generated for various geological parameters viz. lithology, lineaments, geomorphology, slope, land use /land cover, etc. which only dominantly assign the landslide vulnerability grades to the area. These five vector GIS layers were converted into raster layers using ArcGIS (Figs. 6, 7, 8, 9 and 10). Over these five raster GIS layers, the landslide distribution map (Fig. 5) was independently overlaid using Arc GIS software. Based on the number of landslides falling in each subclass of the five geosystem layers, landslides per unit area (weightage) were worked out. This was obtained by dividing the number of landslides falling in each subclass by the total number of pixels of the corresponding subclass. Thus, the weightages were assigned to each subclass or the polygon class of all the five geosystem GIS layers. The data is given in respective tables. The weightage was also assigned to road frequencies. These weighted raster GIS layers were then added using the raster calculator menu of Arc GIS software and thus the final integrated GIS layer was generated with each pixel having the cumulative weightage of all the GIS geosystem layers. Finally, based on the dynamic range of the weightages of the

Table 2: Landslide vulnerability weightages of various classes of lithology (SRB).

\begin{tabular}{|llllll|}
\hline Sr. No. & Lithology & No. of Landslides (LS) & Area or No. of Pixel (A) & (LS /A) & LV weightage (LS/A) × 1000 \\
\hline 1 & Laterite & 15 & 12070 & 0.0012428 & 1.24 \\
2 & Alluvium & 0 & 169 & 0 & 0 \\
3 & Purandargadh weathered basalt & 10 & 6773 & 0.0014765 & 1.48 \\
4 & Diveghat & 9 & 16221 & 0.0005548 & 0.55 \\
5 & Shastri River & 5 & 1882 & 0.0026567 & 2.66 \\
\hline
\end{tabular}

Table 3: Landslide vulnerability weightages of various classes of lineaments (SRB).

\begin{tabular}{|llllll|}
\hline Sr. No. & Lineament Buffer & No. of Landslides (LS) & Area or No. of Pixel (A) & (LS /A) & LV weightage (LS/A) $\times$ 1000 \\
\hline 1 & 400 & 23 & 11555 & 0.0019905 & 1.99 \\
2 & 800 & 10 & 10671 & 0.0009371 & 0.94 \\
3 & 1200 & 4 & 7696 & 0.0005198 & 0.52 \\
4 & 1600 & 1 & 4765 & 0.0002099 & 0.21 \\
5 & 2000 & 0 & 3051 & 0 & 0 \\
\hline
\end{tabular}


Table 4: Landslide vulnerability weightages of various classes of geomorphology (SRB).

\begin{tabular}{|llllll|}
\hline Sr. No. & Geomorphology & No. of Landslides (LS) & Area or No. of Pixel (A) & (LS /A) & LV weightage (LS/A) × 1000 \\
\hline 1 & Deep Valley & 14 & 658663 & 0.000021 & 0.0212 \\
2 & Shallow Valley & 4 & 400006 & 0.000009 & 0.0099 \\
3 & Plain & 1 & 150126 & 0.000006 & 0.0066 \\
4 & Slope & 12 & 43833 & 0.000274 & 0.2737 \\
5 & Ridges & 8 & 766442 & 0.000010 & 0.0104 \\
\hline
\end{tabular}

Table 5: Landslide vulnerability weightages of various classes of slope (SRB).

\begin{tabular}{|llllll|}
\hline Sr. No. & Slope & No. of Landslides (LS) & Area or No. of Pixel (A) & (LS /A) & LV weightage (LS/A) $\times 1000$ \\
\hline 1 & Gentle $\left(0^{\circ}-5^{\circ}\right)$ & 1 & 686389 & 0.00000 & 0.0014 \\
2 & Moderate $\left(6^{\circ}-10^{\circ}\right)$ & 4 & 596648 & 0.00001 & 0.0067 \\
3 & Steep $\left(11^{\circ}-20^{\circ}\right)$ & 33 & 709059 & 0.00005 & 0.0465 \\
4 & Very Steep $\left(21^{\circ}-35^{\circ}\right)$ & 1 & 29376 & 0.00003 & 0.0340 \\
5 & Precipitous $\left(>35^{\circ}\right)$ & 0 & 4553 & 0.00000 & 0 \\
\hline
\end{tabular}

final integrated GIS layer, the study area (SRB) was classified into 5 Landslide vulnerable zones viz. very high, high, moderate, low, and very low (Fig. 11). Details of various steps are discussed in the following paragraphs.

Lithology: SRB is predominantly covered by Deccan Basalts. It has undergone different degrees of weathering. The area has been studied by using tonal difference, drainage density, vegetal coverage from the satellite data and subsequently followed by the field checks. Litho-logically the area is classified into different categories viz. highly weathered, weathered, moderately weathered, poorly weathered, and un-weathered. The zones covered by these classes were digitized into five polygon classes using the screen digitization technique by Arc-GIS. The GIS layer was generated by the conversion of vector to raster form for the lithological studies (Fig. 6).

Lineament density: Based on the tonal, textural, topographical, drainage, and vegetation linearities and curvi-linearities, the fracture-controlled lineaments were observed in IRS- R2 LISS III raw, FCC data, and DEM data. It was mapped and checked in the fields. The buffering technique was adapted to understand the influence of lineaments. For this purpose, five buffer zone classes were marked viz. $400 \mathrm{~m}, 800 \mathrm{~m}, 1200$ $\mathrm{m}, 1600 \mathrm{~m}$, and $2000 \mathrm{~m}$. The GIS database in the form of a raster layer was generated using these five classes with the help of ARC-GIS (Fig. 7).

Geomorphology: For geomorphic studies, the IRS R2 LISS-III data were subjected to various image processing

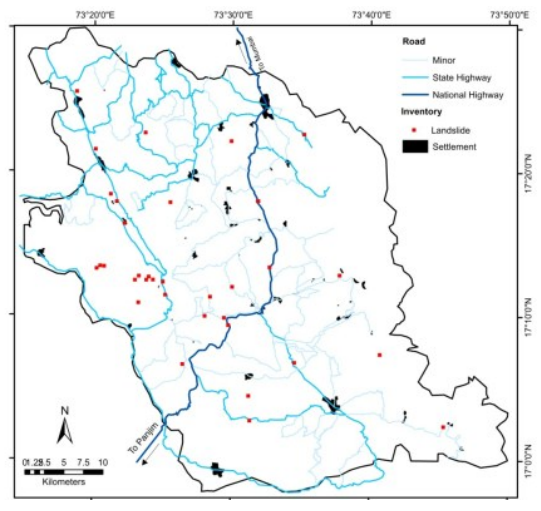

Fig. 5: Landslide inventory and road map of SRB.

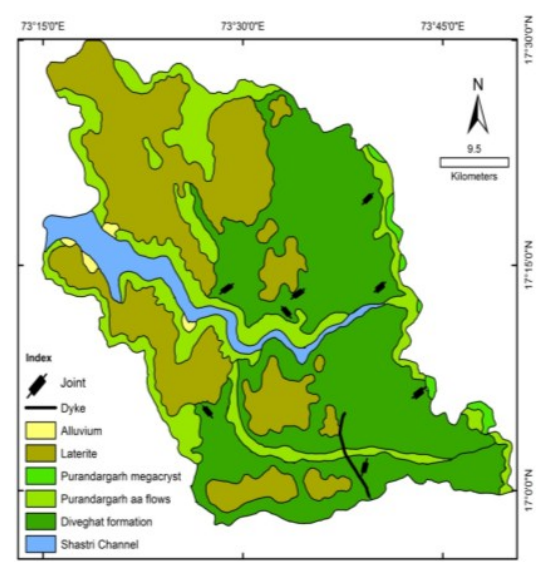

Fig. 6: Lithological map of SRB. 
Table 6: Landslide vulnerability weightages of various classes of LULC (SRB).

\begin{tabular}{|llllll|}
\hline Sr. No. & LULC & No. of Landslides (LS) & Area or No. of Pixel (A) & (LS /A) & LV weightage (LS/A) $\times$ 1000 \\
\hline 1 & Grassy land & 23 & 117890 & 0.0001951 & 0.1951 \\
2 & Water & 0 & 24393 & 0 & 0 \\
3 & Barren (Laterite) & 6 & 431900 & 0.0001389 & 0.0139 \\
4 & Forest & 9 & 528741 & 0.0001722 & 0.0170 \\
5 & Agriculture & 1 & 475596 & 0.0002103 & 0.0021 \\
\hline
\end{tabular}

Table 7: Landslide vulnerability weightages of various classes of roads (SRB).

\begin{tabular}{|llllll|}
\hline Sr. No. & Distance & No. of Landslides (LS) & Area or No. of Pixel (A) & (LS /A) & LV weightage (LS/A) $\times 1000$ \\
\hline 1 & 50 & 7 & 1854 & 0.00377562 & 3.7756 \\
2 & 100 & 4 & 1731 & 0.002310803 & 2.3108 \\
3 & 150 & 1 & 1656 & 0.000603865 & 0.6039 \\
4 & 200 & 1 & 1586 & 0.000630517 & 0.6305 \\
5 & 250 & 8 & 1546 & 0.005174644 & 5.1746 \\
\hline
\end{tabular}

techniques, like contrast stretching, false-color composites, color composites of principal component images, etc. Various geomorphic features considered for the landslide studies viz. deep valleys, plains, ridges, shallow valleys and slopes, etc. along with vegetation were interpreted and vectorized as individual polygon classes. The GIS database was generated by the conversion of the data into the raster layer (Fig. 8).

Slope: To understand the landslide vulnerability zones, the slopes were classified into five categories viz. precipitous (> $\left.35^{0}\right)$, very steep $\left(35^{0}-21^{0}\right)$, steep $\left(11^{0}-20^{0}\right)$, moderate $\left(6^{0}-\right.$ $\left.10^{0}\right)$, and gentle $\left(0^{0}-5^{0}\right)$ (Wentworth, C. K., 1930). The vector GIS layer was generated for these zones showing five polygon classes of slopes and then converted into a raster layer (Fig. 9).

Land use/Land cover: This parameter provides varying degrees of protection and vulnerability to landslides. The natural vegetation and the thick forests anchor the soils and protect the slopes from slope failures, while, the plantations, settlements, highly developed areas with a network of roads, increase the probability of the occurrence of landslides. For this study, the digitally processed IRS R2 LISS -III data were used. The features viz. water bodies, agriculture, forests, built-up, and wasteland were interpreted. They were digitized and a vector GIS layer was generated showing all the features as different polygon classes (Fig. 10).

\section{Assigning Landslide Vulnerability Weightages to Geosystem Parameters}

Generation of raster GIS layers is followed by the assignment

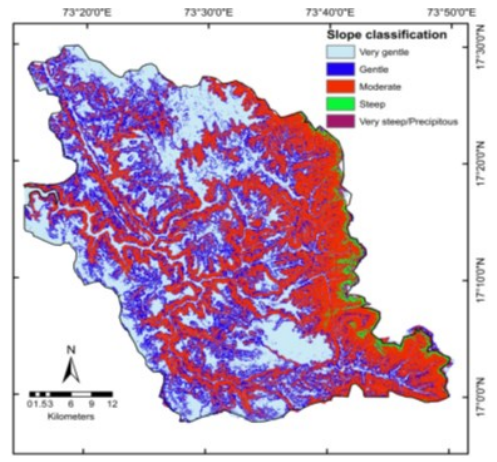

Fig. 9: Slope map of SRB.

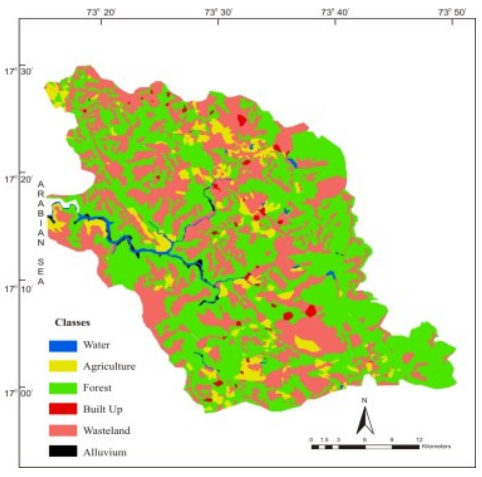

Fig. 10: Land use/Landcover Map of SRB. 
Table 8: Distribution of landslide vulnerable zones (SRB).

\begin{tabular}{|llll|}
\hline Sr. No. & Class & Area or No. of Pixel (A) & Percentage of Area \\
\hline 1 & Very High & 12212 & 28.20 \\
2 & High & 422 & 0.98 \\
3 & Moderate & 10274 & 23.73 \\
4 & Low & 11331 & 26.17 \\
5 & Very Low & 9058 & 20.92 \\
\hline
\end{tabular}

of the landslide vulnerability weightages to each feature class of all the five geosystem layers, based on the number of landslides per unit area. These are raster layers and hence the polygon classes are referred to as the feature classes. The same procedure is done by overlaying the GIS layer of landslides inventory map (Fig. 5) over the above five raster GIS layers of different geosystems individually. It is followed by the counting of the total number of landslides falling in the individual feature class of these five raster layers. It includes counting the total number of pixels in each feature class. The number of landslides (LS) has been divided by the data so obtained, falling in each feature class with the total number of pixels (A) covered by the corresponding feature class. In this step, we assign "weights" to the normalized inputs by multiplying each of them by a value by 1000 and the landslide vulnerability weightages (LVW) assigned to each

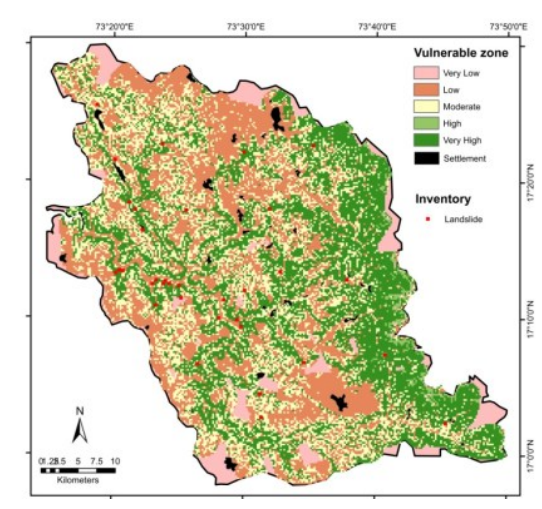

Fig. 11: Landslide vulnerable zone map of SRB.

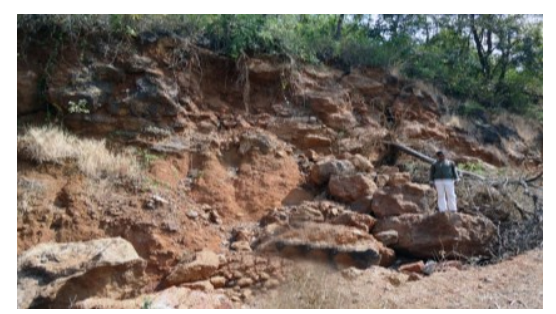

Fig. 12: Rockfall near Bhatgaon - $\left(17^{\circ} 16^{\prime} \mathrm{N}, 7^{\circ} 24^{\prime} \mathrm{E}\right)$. feature class. (Table 2 - Lithology, Table 3 - Lineaments, Table 4- Geomorphology, Table 5-Slope, Table 6 - LULC and Table 7 - road frequencies).

\section{GIS Integration and Landslide Vulnerability}

After assigning the Landslide Vulnerable Weightages (LVW) to the 5 rasterized geosystem layers' feature classes, they were all merged using Raster Calculator. The LVW value of each pixel of the weighted raster layers of all Geo systems and roadways (Fig. 5) was then added and matched, and the final integrated GIS layer representing different landslide vulnerable zones (LVZ) was generated (Fig. 11). In all 43297 pixels, such an integrated GIS output has completely collected LV weightages. Five LVZs have been found based on the LVZ map and data (Table 8), including very high, high, moderate, low, and very low.

\section{CONCLUSION}

The map showing LVZ was prepared by superimposing the landslide inventory layer over the final integrated GIS layer on landslide vulnerability. From the above investigations, it can be concluded that

About 29\% area of the Shastri River Basin (SRB) forms a highly vulnerable zone for land sliding.

These zones are mainly confined to steep slopes $\left(11^{\circ}\right.$ $20^{\circ}$ ), grassy-land, highly weathered basalts (Purandargarh and Diveghat formation) (Figs. 12 and 13), and deep valleys, and in the vicinity of lineaments $(400 \mathrm{~m})$.

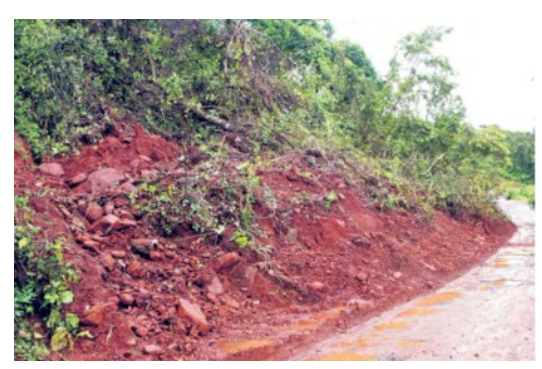

Fig. 13: Debris Slide near Asore (17 $\left.18^{\prime} \mathrm{N}, 7^{\circ} 22^{\prime} \mathrm{E}\right)$. 
Results so obtained were validated in the field using GPS.

\section{ACKNOWLEDGMENT}

Dr. S. B. Joshi is thankful to Dr. S. V. Koti, Principal, Walchand College of Arts and Science, Solapur for extending help during present work and encouragement.

\section{REFERENCES}

Diaz, S., Fargione, J., Chapin, F.S. and Tilman, D. 2005. Biodiversity loss threatens human well-being, PLoS Biol., 4: 1300-1305.

Edison, T, and Ganapathy, P. 2020. Evaluation of landslide hazard and its impacts on the hilly environment of the Nilgiris District - a geospatial approach. Geoenviron. Disasters, 7(3): 139. https://doi.org/10.1186/ s40677-019-0139-3

Greenbaum, D., Tutton, M., Bowker, M., Browne, T., Bulekha, J., Greally, K., Kuna, G., McDonald, A., Marsh, S., O'Connor, E. and Tragheim, D. 1995. Rapid methods of landslide mapping: Papua New Guinea case study. British Geol. Sur., Keyworth, Nottingham, UK, Tech. Report WC/95/27, p. 121

Guzzetti, F., Mondini, A.C., Cardinali, M., Fiorucci, F., Santangelo, M. and Chang, K.T. 2012. Landslide inventory maps: new tools for an old problem. Earth Sci. Rev., 112: 42-66. https://doi.org/10.1016/j. earscirev.2012.02.001
Jiu, J.J., Xu-Sheng, W. and Subhas, N. 2005. Confined groundwater zone and slope instability in weathered igneous rocks in Hong Kong. Eng. Geol., 80: 71-92

Juang, C.S., Stanley, T.A. and Kirschbaum, D.B. 2019. Using citizen science to expand the global map of landslides: introducing the cooperative open online landslide repository (COOLR). PLoS One, 14: e0218657

Meena, S.R., Ghorbanzadeh, O. and Hölbling, D. 2019. Comparison of Event-Based landslide inventories: A case study from Gorkha earthquake 2015, Nepal. Paper presented at the European Space Agency's 2019 Living Planet Symposium, Milan, Italy, 13-17 May 2019, European Space Agency, Paris, France, pp. 1-18.

Mitchell, C. and Cox, K.G. 1988. A geological sketch map of southern part of the Deccan province. Memoir of Geol. Soc. India, 10: 27-33

Nagarajan, R., Mukharji, A., Roy, A. and Khaire, M.V. 1998. Temporal remote sensing data and GIS application in landslide hazard zonation of part of Western Ghat, India. Int. J. Remote Sens., 19(4): 573-585

Prakash, N., Manconi, A. and Loew, S. 2020. Mapping landslides on EO data: performance of deep learning models vs. traditional machine learning models. Remote Sens., 18: 1937-1950

Ramakrishnan, S.S., Sanjeevi Kumar V., Zaffar Sadiq, M.G.S.M., Arulraj, M. and Venugopal, K. 2002. Landslide disaster management and planning: A GIS-based approach. Indian Cartog., 05: 192-195

Valdiya, K.S. 2011. Some geodynamic hot spots in India require urgent comprehensive studies. Curr. Sci., 100 (10): 1490-1499

Van Westen, T.W.J. Van, A. and Soeters, R. 2006. Landslide hazard and risk zonation: Why is it so difficult? Bull. Eng. Geo. Env., 65(2): 167-184

Wentworth, C.K.1930. A simplified method of determining the average slope of land surfaces Am. J. Sci., 21: 184-194 